\title{
COLABORACIÓN ESPECIAL
}

\section{NUTRIGENÓMICA, OBESIDAD Y SALUD PÚBLICA}

\author{
Manuela-Belén Silveira Rodríguez, Lourdes Martínez-Piñeiro Muñoz y Raffaele Carraro Casieri.
}

Unidad de Obesidad. Servicio de Endocrinología y Nutrición. Hospital Universitario de La Princesa. Madrid.

\section{RESUMEN}

El desarrollo de la genómica funcional en los próximos años condicionará cambios en el conocimiento teórico y la práctica clínica de la nutrición. La posibilidad de determinar el perfil genético de un individuo (variaciones genéticas y modificaciones epigenéticas) y de conseguir la integración de estos datos en una compleja red de interacciones metabólicas constituye un desafío sin precedentes en la nutrición humana. La aplicación práctica del conocimiento científico puro derivado de la nutrigenómica, en términos de prevención y tratamiento de la obesidad, la DM2 y las enfermedades cardiovasculares, y sus implicaciones en la salud pública, son en este momento todavía indeterminadas. La posibilidad de una intervención nutricional en periodos críticos del desarrollo y la capacidad de modificar la susceptibilidad genética a ciertas enfermedades a través de la alimentación es el gran reto de la nutrigenómica, más allá del diseño de dietas o alimentos funcionales "personalizados".

Palabras clave: Genes. Genómica. Epistasia Genética. Nutrigenómica. Obesidad. Enfermedad cardiovascular. Salud pública. Nutrición. Epigenética.

\section{ABSTRACT \\ Nutrigenomics, Obesity and Public Health}

Functional genomics will change knowledge and practice in clinical nutrition in the forthcoming years. The possibility of performing an individual's genetic profile (genetic variations and epigenetic modifications) as well as the ability of its integration in a complex network of metabolic interactions represents a huge challenge in Human Nutrition. The influence of nutrigenomics in terms of prevention and treatment of chronic diseases, such as obesity, type 2 diabetes and cardiovascular disease in a population level remains undetermined for the moment. The opportunity of nutritional intervention in critical stages of development and the chance of changing genetic susceptibility to diseases through diet in a Public Health basis should lead the future of nutrigenomics beyond the mere design of "personalized" functional food or diets.

Key words: Genes. Genomics. Epistasis, Genetic. Obesity. Cardiovascular diseases. Public health. Nutrition. Epigenetic. Nutrigenomics.

\footnotetext{
Correspondencia:

Manuela-Belén Silveira Rodríguez

Unidad de Obesidad

Servicio de Endocrinología y Nutrición

Hospital Universitario de La Princesa

C/ Diego de León, 62. 28006 Madrid

Correo electrónico: HYPERLINK "mailto:belensilvei-

ra@telefonica.net" belensilveira@telefonica.net
} 


\section{INTRODUCCIÓN}

La obesidad es una enfermedad crónica caracterizada por el incremento suprafisiológico de los depósitos grasos del organismo ligada a una constelación de patologías asociadas, tales como la diabetes mellitus tipo 2 (DM2), la dislipemia, la hipertensión arterial y la enfermedad cardiovascular, que finalmente determinan una disminución de la expectativa de vida ${ }^{1}$. Este incremento de la morbimortalidad comienza a evidenciarse cuando la relación entre el peso y talla al cuadrado de un individuo (índice de masa corporal, $\mathrm{IMC}=\mathrm{Kg} / \mathrm{m}^{2}$ ) es superior a 25 $\mathrm{Kg} / \mathrm{m}^{2}$ (sobrepeso) y especialmente a partir de un IMC de $30 \mathrm{Kg} / \mathrm{m}^{2}$ (obesidad) ${ }^{1,2}$. Más allá de las consecuencias sobre la calidad de vida y la supervivencia del individuo, el impacto socioeconómico en los próximos años de una población como la española con una prevalencia de exceso ponderal (sobrepeso y obesidad) de al menos el 50\% ${ }^{3}$ es incalculable. En la tabla 1 se muestra la prevalencia de obesidad (IMC $\geq 30 \mathrm{Kg} / \mathrm{m}^{2}$ ) en España según grupos de edad y sexo ${ }^{3-5}$.
La aparición de la obesidad en un individuo puede entenderse como la resultante de la interacción entre su dotación genética y las influencias ambientales, entre las cuales se encuentran el nivel de actividad física y sus elecciones alimentarias, tanto en cantidad (aporte energético) como en calidad (nutrientes y otros componentes de los alimentos). El número de genes implicados en la regulación de la homeostasis energética y el apetito, el peso corporal y la adiposidad, con posible trascendencia en la etiología de la obesidad, es enorme. El mapa genético de la obesidad involucra al menos 600 genes, marcadores y regiones cromosómicas ("http://obesitygene.pbrc.edu/"), con presencia en el ser humano de posibles loci relacionados con un fenotipo obeso en todos los cromosomas excepto el Y. Los principales loci relacionados con la obesidad se encuentran en los cromosomas 4, 10 y 20. Se han descrito múltiples mutaciones en un solo gen asociadas a la obesidad, de las cuales los genes en los que se han

Tabla 1

Prevalencia de obesidad en España según grupos de edad y sexo

\begin{tabular}{|c|c|c|c|}
\hline Población infanto-juvenil* & $\begin{array}{c}\text { Hombres } \\
\text { (\% prevalencia) }\end{array}$ & $\begin{array}{c}\text { Mujeres } \\
\text { (\% prevalencia) }\end{array}$ & $\begin{array}{c}\text { Total } \\
\text { (\% prevalencia) }\end{array}$ \\
\hline 2-9 años & 16,3 & 11,6 & 14,0 \\
\hline 10-17 años & 18,5 & 9,1 & 13,9 \\
\hline 18-24 años & 12,6 & 14,9 & 13,7 \\
\hline \multicolumn{4}{|l|}{ Población adulta $\uparrow$} \\
\hline 25-34 años & 7,1 & 4,8 & 5,9 \\
\hline 35-44 años & 11,7 & 12,2 & 12,0 \\
\hline 45-54 años & 16,9 & 26,4 & 22,0 \\
\hline 55-64 años & 21,5 & 34,2 & 28,5 \\
\hline \multicolumn{4}{|l|}{ Población anciana (65 años y más) } \\
\hline Institucionalizados & 20,5 & 21,8 & 21 \\
\hline No institucionalizados $\S$ & 31,5 & 40,8 & 36 \\
\hline
\end{tabular}

* Estudio EnKid (IMC > P97 para edad y sexo, tablas de Orbegozo, Hernández et al).

Estudio DORICA (IMC $\geq 30 \mathrm{Kg} / \mathrm{m}^{2}$ ).

$\doteqdot$ Aranceta et al $\left(\mathrm{IMC} \geq 30 \mathrm{Kg} / \mathrm{m}^{2}\right)$.

$\S$ Gutiérrez-Fisac et al $\left(\mathrm{IMC} \geq 30 \mathrm{Kg} / \mathrm{m}^{2}\right)$. 
replicado el mayor número de estudios son precisamente aquellos implicados en el metabolismo energético, la adipogénesis y la señalización celular (peroxisome proliferator -activated receptors $\gamma$, PPARG; guanine nucleotide binding protein ( $G$ protein), beta polypeptide 3 , GNB3), genes que codifican para adipocitoquinas y otros factores involucrados en la regulación del apetito y la ingesta (leptina, LEP, y su receptor, LEPR; adiponectina, ADIPOQ; receptor 2c de 5ahidroxitriptamina, HTR2C; nuclear receptor subfamily 3 , group c, member 2, NR3C1, también denominado receptor de mineralcorticoides; receptor tipo 4 de melanocortina, MCR4; proopiomelanocortina, POMC), genes involucrados en la regulación de la termogénesis y el gasto energético (proteínas desacopladoras mitocondriales, UCP1, UCP2 y UCP3, genes de la familia de los receptores adrenérgicos $\beta$, ADRB3, ADRB2 ${ }^{6}$ y otros con función no bien conocida ( $f a t$ mass and obesity associated gene, $\mathrm{FTO}^{7}$ ) A nivel poblacional, las obesidades monogénicas constituyen un pequeñísimo porcentaje (OMIM, Online Mendelian Inheritance in Man; http:// www.ncbi.nlm.nih.gov/entrez/dispomim.cgi?id=601665). Más aún, en algunos casos, la combinación precisa en un individuo de alelos específicos de 2 genes (o rara vez más de 2) provoca la aparición de un determinado fenotipo. Esta interacción gen-gen se denomina epistasia o epistasis y por el momento en el ser humano se están empezando a describir ciertas interacciones epistáticas relacionadas con la obesidad (genotipos específicos de TBC1D1, una proteína que contiene un dominio que puede funcionar como activador de GTPasa). Sin embargo, en la mayoría de los individuos que serán obesos (obesidad "esporádica" poligénica) se establece una compleja red de interacciones difícil de estudiar entre genes y medio ambiente (estilo de vida) donde los diferentes patrones alimentarios desempeñan un papel relevante, más allá del simple aporte de un exceso de energía.

\section{INTERACCIONES ENTRE GENES Y NUTRIENTES: LA GENÓMICA FUNCIONAL}

El estudio de la interacción entre genes y nutrientes, reciente y vertiginoso, conllevará cambios en la investigación y la práctica de la nutrición humana, tanto a nivel individual como poblacional.

La nutrigenética desarrolla el conocimiento científico que explica el impacto de las variaciones genéticas individuales en los requerimientos óptimos de un determinado nutriente para un determinado sujeto, frente a los principios tradicionales en nutrición basados en recomendaciones de ingesta a nivel poblacional con base epidemiológica y resumidos en conceptos como las Recommended Dietary Allowances (RDAs) o nivel medio diario de consumo de un nutriente suficiente para cubrir requerimientos del $97-98 \%$ de la población sana de una determinada edad, sexo y etapa de la vida.

La nutrigenómica es la disciplina que estudia la regulación de los genes por la dieta (su función: transcripción y translación, y metabolismo). Así, la transcriptómica (transcriptoma: colección completa de tránscritos de ácido ribonucléico, ARN, producido por el ácido desoxirribonucléico, ADN, de un genoma), la proteómica (proteoma: conjunto total de proteínas derivadas del transcriptoma, incluyendo sus ulteriores modificaciones) y la metabolómica (metaboloma: la suma total de metabolitos endógenos y exógenos, p.ej los derivados de la flora colónica) constituyen la denominada genómica funcional.

Las interacciones entre genes y nutrientes son complejas y variadas. Existen 3 
grandes grupos conceptuales: variaciones genéticas y necesidades de nutrientes, interacciones directas nutriente-gen e interacciones epigenéticas (tabla 2).

\section{VARIACIONES GENÉTICAS Y REQUERIMIENTOS DIETÉTICOS}

El código genético de individuos no emparentados coincide en un 99,9\%. Los polimorfismos son lugares del ADN donde frecuentemente difieren las secuencias en distintos individuos (al menos en un $1 \%$ de la población). Los más frecuentes SNPs (single nucleotide polymorphisms) son cambios de una sola letra en el código (un solo nucletótido); un individuo puede llevar diversas combinaciones de un determinado polimorfismo ( 2 copias de cada gen). El genotipo es la combinación de secuencias en las 2 copias de un gen para un polimorfismo particular. Se han identificado más de 10 millones de SNPs, de los cuales los más comunes aparecen en un 5 hasta un $50 \%$ de la población. La mayoría de los humanos somos heterocigotos para más de 50.000 SNPs en nuestros genes, muchos de los cuales implican una alteración en la expresión génica o cambios en la estructura o función de sus productos (proteínas). El análisis de todos los SNPs de un individuo es actualmente impracticable. Sin embargo, el hecho de que los SNPs cercanos en la secuencia de ADN de un gen tiendan a heredarse juntos (haplotipo) y que la mayoría de las regiones cromosómicas solo tiene unos cuantos haplotipos comunes, permite de forma práctica el análisis de éstos y de agrupaciones de $\mathrm{SNPs}^{8}$ (http://hapmap.org).

Los SNPs son parte de los mecanismos de adaptación al entorno en la evolución humana y condicionan la diversidad poblacional, la individualidad, la susceptibilidad a ciertas enfermedades y asimismo la idiosincrasia en las respuestas a fármacos. Algunos SNPs comunes en la población determinan para los sujetos portadores requerimientos especiales de nutrientes. Por ejemplo, la 5, 10-metilentetrahidrofolatoreductasa (MTHFR) es una enzima implicada en el metabolismo del ácido fólico cuya variante termolábil (homocigosis C677T, presente en el 5 hasta el $30 \%$ de la población) presenta una reducción de su actividad y se relaciona con un incremento de las concentraciones de homocisteína en plasma (figura 1) y un incremento del riesgo de enfermedad cardiovascular $^{9,10}$ y tromboembólica ${ }^{11,12}$ y aumento de complicaciones obstétricas en algunos estudios ${ }^{13,14}$. La ingesta de elevadas cantidades de folatos consigue una normalización de la concentración de homocisteína en plasma, aunque su repercusión en términos de morbilidad cardiovascular está por demostrar ${ }^{15-19}$.

El gen APOA1 (apoproteína A1, componente fundamental del colesterol HDL) es

Tabla 2

Interacciones entre genes y nutrientes

\begin{tabular}{|l|l|l|}
\hline \multicolumn{1}{|c|}{ Gen } & \multicolumn{1}{|c|}{ Nutriente } & \multicolumn{1}{c|}{ Ejemplo } \\
\hline Variación genética (SNP) & Condicionamiento de una necesidad particular & $\begin{array}{l}\text { Homocigosis C677T MTHFR: incremento de } \\
\text { las necesidades de folatos }\end{array}$ \\
\hline Modificación aguda de la expresión génica & Interacción directa & PPARs y ácidos grasos poliinsaturados \\
\hline Modificación crónica de la expresión génica & Regulación epigenética & $\begin{array}{l}\text { Modificaciones nutricionales en periodos crí- } \\
\text { ticos del desarrollo }\end{array}$ \\
\hline
\end{tabular}

SNP: single nucleotide polymorphism.

MTHFR: 5, 10-metilentetrahidrofolatoreductasa.

PPARs: peroxisome proliferator -activated receptors. 
altamente polimórfico. Dependiendo del genotipo de una determinada región promotora (APOA1-75G $\rightarrow$ A), la ingesta baja (homocigosis para el alelo $G$ ) o elevada (portadores del alelo A) de ácidos grasos poliinsaturados determina un incremento en las concentraciones de colesterol HDL en mujeres ${ }^{20}$.

Los receptores PPAR regulan más de 300 genes, muchos de ellos involucrados en el metabolismo lipídico extracelular y oxidación de ácidos grasos. A su vez, los PPAR están regulados por ácidos grasos y metabolitos de ácidos grasos, que se comportan como ligandos de los PPAR $\alpha$. Así, otro ejemplo de variación genética determinante de una diferente necesidad de nutrientes es un SNP en el gen del PPAR $\alpha$ (PPARA Leu162Val) asociado con alteraciones en el colesterol total, colesterol LDL y apoproteína B que determina en portadores del alelo V162 una marcada reducción en la concentración de triglicéridos en respuesta a ácidos grasos poliinsaturados ${ }^{20}$.

Los ejemplos previamente descritos ilustran cómo ciertos SNPs pueden determinar una concreta recomendación al individuo en cuanto a su alimentación pero también muestran la complejidad de las relaciones entre genes, nutrientes y rutas metabólicas. De este modo, una portadora del alelo V162 en PPARA a la que se le recomendara una elevada ingesta de ácidos grasos poliinsaturados para disminuir sus cifras de triglicéridos podría padecer como efecto adverso un descenso de su colesterol HDL en caso de ser homocigota para el alelo G de APOA1.

\section{INTERACCIONES DIRECTAS ENTRE GENES Y NUTRIENTES}

Son las interacciones conocidas desde hace más tiempo, en las cuales un nutriente se comporta básicamente como un factor de transcripción que modifica de forma aguda la expresión génica ${ }^{21}$. Son ejemplos clásicos las interacciones con sus receptores nucleares de la vitamina $\mathrm{D}$ o los derivados retinoicos de la vitamina A. Como previamente se ha mencionado, los ácidos grasos de la dieta ${ }^{22,23}$ (especialmente ácidos grasos mono y poliinsaturados) son capaces de ligarse directamente a los receptores PPAR y modificar la expresión de otros genes críticos involucrados en el metabolismo lipídico (NR1H2/LXR, liver X receptor) o disminuir la disponibilidad de la sterol regulatory element-binding protein-1, SREBP-1 (inhibición de su activación proteolítica por ácidos grasos poliinsaturados, fundamentalmente omega 3 ) un regulador de genes que codifican para proteínas involucradas en la síntesis del colesterol y la lipogéne$\mathrm{sis}^{24}$. Es decir, los ácidos grasos se comportan como factores de transcripción críticos para la expresión de una colección de genes involucrados en el metabolismo lipídico y el proceso de adipogénesis ${ }^{25}$.

Una muestra de la aplicación en la industria alimentaria de estos conceptos son los alimentos funcionales con ácido linoléico

Figura 1

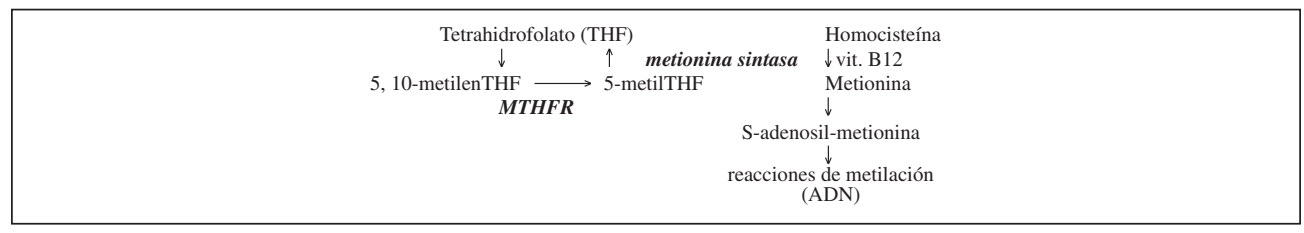

A partir del folato ingerido con la alimentación, el tetrahidrofolato se comporta como un aceptor/donador de grupos metilo, proceso en el que se encuentra involucrada la enzima clave metilentrahidrofolatoreductasa (MTHFR). Este ciclo se halla imbricado con la síntesis de purinas y pirimidinas (no reflejado en el esquema) y con los fenómenos de metilación del ADN a través de la acepción por la homocisteína de un grupo metilo donado por el 5-metilTHF, reacción catalizada por la metionina sintasa. La variante termolábil de la MTHFR (C677T) presenta una actividad reducida e induce, en condiciones dietéticas de escasez de folatos, un incremento de los niveles plasmáticos de homocisteína y un incremento del riesgo cardiovascular. 
conjugado (CLA), un tipo de ácido graso con supuestos efectos beneficiosos sobre el peso y la composición corporal (aumento de la masa magra y disminución de la masa grasa). En realidad, los CLAs son un conjunto de isómeros posicionales y geométricos del ácido linoléico con enlaces conjugados dienóicos, de los cuales aquéllos con actividad biológica conocida son el CLA cis-9, trans-11 y trans-10, cis-12. En concreto, el isómero trans10,cis-12 ha demostrado, en ratones y otros animales, poseer la capacidad de disminuir el peso y modificar la composición corporal ${ }^{26}$, aunque en humanos los resultados son contradictorios ${ }^{27-30}$. Los posibles mecanismos inductores de sus efectos sobre la composición corporal no están totalmente aclarados, pero se sabe que CLA trans-10, cis-12 es capaz de modificar la expresión de diversos genes (PPARs, SREBP-1) implicados en la adipogénesis y en la actividad de diversas enzimas involucradas el metabolismo energético ${ }^{31-34}$. Sin embargo, algunos autores han comunicado la posibilidad de acciones desfavorables del CLA trans10, cis-12 sobre el perfil lipídico y la sensibilidad a la insulina ${ }^{35-37}$. Es destacable que el CLA cis-9, trans-11, el isómero presente primordialmente de forma natural en leche y carne de rumiantes, parece ejercer acciones antiinflamatorias a nivel de tejido adiposo y promover un aumento de la sensibilidad a la insulina ${ }^{38,39}$. La proporción de los diferentes isómeros es variable en los preparados comerciales destinados a la alimentación humana y por tanto parece prudente la precaución en su empleo, especialmente en pacientes obesos diabéticos o con síndrome metabólico, una considerable fracción de la población de obesos ${ }^{40}$.

\section{REGULACIÓN EPIGENÉTICA Y NUTRICIÓN}

\section{El epigenoma}

La expresión génica puede verse modificada a lo largo de la vida de un individuo sin necesidad de alteraciones en la secuencia de ADN por medio de los llamados cambios epigenéticos, fundamentalmente a través de de la metilación del ADN y la acetilación y metilación de histonas. Estos patrones de modulación de la expresión génica (represión o expresión) mantenidos de forma estable a lo largo del tiempo e incluso con capacidad de ser transmitidos transgeneracionalmente ${ }^{41}$ constituyen el denominado epigenoma. La regulación epigenética permite la expresión o represión estable de genes en las diversas líneas celulares y en los distintos estadios del desarro1lo. La inactivación de uno de los cromosomas $\mathrm{X}$ en las células somáticas femeninas y el silenciamiento alélico del imprinting o sellado genómico, que determina que en ciertos genes su expresión dependa de si son heredados del padre o la madre, constituyen ejemplos clásicos de regulación epigenética. Estas modificaciones epigenéticas precisan una compleja maquinaria que permite que se mantengan en los sucesivos ciclos celulares de tal forma que se preserve el epigenoma. Además de factores intrínsecos (metiltransferasas) otros factores ambientales y nutricionales inciden en la regulación epigenética. La modificación en la disponibilidad de grupos metilo a través de la alimentación puede determinar cambios en la metilación de genes, la modificación de su expresión y transformaciones en el fenotipo, especialmente durante el desarrollo fetal y ciertos periodos críticos del desarrollo.

\section{Interacciones epigenéticas en periodos críticos del desarrollo}

Diversos estudios epidemiológicos ${ }^{42,43}$ $\mathrm{y}$ estudios in vivo en animales muestran cómo la malnutrición materna durante la gestación desencadena una serie de adaptaciones metabólicas fetales ("fenotipo ahorrador") $)^{44}$ que en la edad adulta aumentan el riesgo de desarrollar enfermedades cardiovasculares ${ }^{45-49}$, hipertensión 
$\operatorname{arterial}^{50}$, obesidad ${ }^{51}$, hiperinsulinemia e hiperleptinemia ${ }^{52}$ y resistencia a la insulina y DM2 $2^{53-55}$, especialmente en condiciones de sobreaporte energético ${ }^{56}$. Aunque los mecanismos no están claros, las modificaciones epigenéticas de la expresión génica parecen desempeñar un papel importante. Hipotéticamente, el entorno nutricional fetal adapta al feto para sobrevivir a largo plazo en un entorno postnatal de similares características. Cuando este entorno difiere del de la vida adulta (predicción errónea), las adaptaciones fetales resultan inapropiadas y como consecuencia se desarrolla la enfermedad (Predictive Adaptative Response Hypothesi ${ }^{57}$ ). Así, un feto que se desarrolla en un entorno nutricional adverso se adapta metabólicamente (programación o imprinting metabólico) de tal modo que en el futuro, en condiciones de sobreaporte energético, emergen la obesidad y el síndrome metabólico como consecuencia de una inadecuación con el entorno nutricional existen$\mathrm{te}^{58}$. Estas respuestas adaptativas ocurren en períodos críticos del desarrollo, distintos en los diferentes órganos, durante el periodo fetal y postnatal.

Por tanto, es posible que existan ciertas ventanas temporales en las que modificaciones nutricionales originen cambios permanentes (programación o imprinting nutricional). Por ejemplo, la leptina, una adipoquina implicada en la regulación central del apetito y el balance energético al actuar como una señal de los depóstitos grasos del organismo, posee también efectos tróficos sobre ciertas neuronas hipotalámicas involucradas en la respuesta a nutrientes y contribuye a su plasticidad en el periodo neonatal pre$\mathrm{coz}^{59,60}$. La leptina pudiera también regular durante este periodo la proliferación de células beta pancreáticas ${ }^{61}$. Más aún, la administración subcutánea de leptina recombinante en el periodo neonatal precoz a las crías de ratas malnutridas durante la gestación es capaz de revertir la programación metabólica fetal a un fenotipo normal en la edad adulta ${ }^{62}$. En humanos, se ha encontrado asimismo una correlación negativa entre el IMC a los 2 años de edad y la concentración de leptina en leche materna de madres no obesas, a su vez dependiente del grado de adiposidad materna ${ }^{63}$. Otros estudios no han encontrado esta relación ${ }^{64,65}$. La leptina pudiera ser uno de los múltiples componentes bioactivos de la leche materna que explican el hallazgo en algunos estudios ${ }^{66-68}$ (no en $\operatorname{todos}^{69,70}$ ) de una menor prevalencia de obesidad en edad adulta de los lactantes amamantados frente a aquellos alimentados con leche de fórmula.

La inducción de cambios epigenéticos por la alimentación es también posible en otras etapas del desarrollo. Se ha encontrado mayor supervivencia y menor riesgo de DM en los nietos de aquellos abuelos paternos que sufrieron escasez de alimentos en el periodo de crecimiento lento previo a la pubertad $^{71}$. En este periodo emerge el primer pool viable de espermatocitos $y$ comienza la reprogramación del imprinting en ciertos genes. Curiosamente el gen del factor de crecimiento similar a la insulina tipo 2 (IGF-2) se expresa únicamente en el alelo paterno en la mayoría de tejidos durante el desarrollo fetal y tras el nacimiento $^{72,73}$. Al igual que IGF-2, involucrado en el desarrollo placentario y el control del aporte de nutrientes al feto, otros genes sellados, o imprinted genes, se expresan en placenta y regulan la demanda fetal de nutrientes y también se expresan en áreas del cerebro, como el hipotálamo, involucradas en el control del apetito y la homeostasis energética. Las variaciones epigenéticas en respuesta a nutrientes en estos genes es probable que hayan desempeñado un papel importante en la evolución. Más aún, ciertos síndromes asociados a alteraciones en el imprinting, como el síndrome de PraderWilli, asocian característicamente obesidad y DM2.

Los gemelos monocigóticos discordantes en cuanto a IMC en edad adulta son 
raros pero representan una oportunidad única de estudiar los cambios epigenéticos potencialmente involucrados en la obesidad, así como las ventanas temporales en las que las modificaciones nutricionales puedan ser relevantes. En un estudio realizado en gemelos monocigóticos finlandeses, aquéllos con un IMC diferente en edad adulta ya manifestaban un fenotipo similar (diferencias de peso significativas) al nacimiento; estas diferencias se atenuaron a los 6 meses de vida pero comenzaron a reaparecer en la adolescencia y curiosamente a los 8 años edad (periodo de crecimiento lento prepuberal) se encontraban todos por encima del peso esperado en caso de parto no gemelar ${ }^{74}$.

\section{Alteraciones epigenéticas, envejecimiento y obesidad: alimentación y preservación del epigenoma}

Entendiendo la regulación epigenómica como una adaptación al entorno, es por tanto imprescindible la preservación del epigenoma a lo largo de la vida. La influencia de la alimentación en este sentido no se limita a las acciones directas de los nutrientes presentes en los alimentos (colina, ácido fólico, vitamina B6, B12) (figura 1) sobre la conservación de los patrones de metilación epigenéticos. Otros componentes (aditivos, pesticidas, tóxicos) pueden ser capaces de producir alteraciones en la metilación del ADN. Por ejemplo, un antifúngico frecuentemente empleado en la viticultura, la vinclozolina, posee acciones antiandrogénicas y, comportándose como un disruptor endocrino, potencialmente pudiera generar patrones anómalos de metilación del ADN e inducir alteraciones en la fertilidad y cáncer también en humanos ${ }^{75}$.

Sin embargo, tanto por influencias medioambientales como debido a fenómenos estocásticos, con el paso del tiempo se acumulan alteraciones epigenéticas que implican modificaciones en el fenotipo. La acumulación de errores en la metilación del ADN y la pérdida de regulación epigenética están involucrados en los procesos de tumorogénesis y envejecimiento ${ }^{76}$. Más aún, una vez que aparecen patrones aberrantes en la regulación epigenética, no sólo es posible que éstos se mantengan a lo largo de la vida del sujeto, sino que sean heredados en las siguientes generaciones ${ }^{71,77}$. Quizás la alteración en la regulación epigenética, potencialmente transmitida de padres a hijos y a nietos, sea una de las claves que expliquen el aumento de la prevalencia de la obesidad en las últimas generaciones.

Las modificaciones en la expresión génica que se producen en adipocitos envejecidos ${ }^{78}$ implican alteraciones en las diversas rutas metabólicas, en la secreción de adipoquinas y en la capacidad de hiperplasia análogas a las que se producen en el tejido adiposo funcionalmente saturado por un sobreaporte energético crónico. Entre ambas situaciones, obesidad y envejecimiento, se puede establecer un paralelismo funcional en el tejido adiposo. Así, la obesidad constituye un estado de disfunción adipocitaria. Es decir, implica tanto un agotamiento de la capacidad de almacenamiento de ácidos grasos (disminución de la adipogénesis; incremento de la apoptosis) como una perturbación en la función endocrinometabólica del tejido adiposo ${ }^{79}$. Estos sucesos generan un incremento relativo del tejido adiposo visceral (obesidad visceral) y, en último término, la aparición de depósitos grasos aberrantes o ectópicos. La consecuencia, en términos fisiopatológicos, es la inducción de unos cambios adaptativos en la homeostasis corporal que mantenidos de forma crónica acaban por ocasionar alteraciones endocrinometabólicas (lipotoxicidad y lipoapotosis ${ }^{80,81}$, incremento de la insulinorresistencia e hiperinsulinemia compensadora ${ }^{82}$ ) y la generación de un estado inflamatorio de bajo grado $^{83}$, proaterogénico ${ }^{84}$, protrombótico y procoagulante. El desenlace es la 
aparición de una constelación de patologías asociadas (DM2, dislipemia, hipertensión arterial, enfermedad cardiovascular, etc) y una disminución de la expectativa de vida.

\section{CONCLUSIONES}

El desarrollo de la genómica funcional en los próximos años condicionará cambios en el conocimiento teórico y la práctica clínica de la nutrición. La posibilidad de determinar el perfil genético de un individuo (variaciones genéticas y modificaciones epigenéticas) y de detectar miles de metabolitos endógenos y exógenos en una muestra biológica y conseguir la integración de estos datos en una compleja red de interacciones metabólicas constituye un desafío sin precedentes en la Nutrición Humana. En Europa, la red NuGO (NutriGenomics Organization, 'The European Nutrigenomics Organisation: linking genomics, nutrition and health research'), una Red europea de Excelencia, desarrolla diversos proyectos de colaboración encaminados a la integración del conocimiento y tecnologías post-genómicas. Financiado por la Unión Europea, destaca el Early Nutrition Programming Project (EARNEST), cuyos objetivos son la identificación de intervenciones capaces de prevenir y revertir una programación nutricional temprana adversa y la mejora de las fórmulas de leche adaptada. Asimismo son precisos grandes estudios poblacionales, como los programas NUGENOB (http://www. nugenob.com) y Diogenes (http://www. diogenes-eu.org), que permitan el estudio de las interacciones entre genes y nutrientes y la identificación de los determinantes genéticos susceptibles de influencias medioambientales que incidan en el desarrollo de la obesidad.

La capacidad no sólo de adaptar el consumo de nutrientes a las necesidades exigi- das por el particular bagaje genético de un individuo (variaciones genéticas) para evitar enfermedades y mejorar su calidad de vida, sino de modular la expresión génica mediante modificaciones nutricionales con efecto no únicamente agudo (interacciones directas gen-nutriente) sino permanente a lo largo de la vida del individuo y, más aún, con la posibilidad de transmitir estos patrones de expresión génica a la descendencia (regulación epigenética) constituye el gran reto de la nutrigenómica. Las enfermedades crónicas, de causa multifactorial en las que la alimentación condiciona, al menos en parte, su desencadenamiento y severidad, son un campo teórico en el que la nutrigenómica potencialmente desempeñará un papel fundamental. Sin embargo, las aplicaciones prácticas del conocimiento científico puro derivado de la genómica funcional, en términos de prevención y tratamiento de la obesidad, la DM2 y las enfermedades cardiovasculares, así como sus implicaciones en la salud pública, son en este momento todavía indeterminadas.

La posibilidad de una intervención nutricional en periodos críticos del desarrollo (preconcepcional, gestacional, postnatal, infantil) que determine una disminución del riesgo de padecer enfermedades como la obesidad en edad adulta o la capacidad de modificar la expresión génica a través de la alimentación y con ello influir en diversos factores de riesgo cardiovascular y en la susceptibilidad genética a ciertas enfermedades, son unos objetivos ambiciosos en nutrición y salud pública, más allá de la repercusión de la nutrigenómica en la nutrición del individuo a través del diseño de dietas o alimentos funcionales "personalizados". El desarrollo de la nutrigenómica implicará inevitablemente la consideración de ciertos aspectos éticos (autonomía, consentimiento informado, privacidad y acceso a la información, equidad) y la necesidad de su regulación legal. 


\section{BIBLIOGRAFÍA}

1. Rubio MA, Salas-Salvadó J, Barbany M, et al. Consenso SEEDO 2007 para la evaluación del sobrepeso y la obesidad y el establecimiento de criterios de intervención terapéutica. Rev Esp Obes. 2007; 5 (3): 135-75.

2. Calle EE, Thun MJ, Petrelli JM, et al. Body mass index in a prospective cohort of U.S. adults. N Engl J Med. 1999; 341: 1097-105.

3. Aranceta-Bartrina J, Serra-Majem Ll, Foz-Sala M, et al. Prevalencia de obesidad en España. Med Clin (Barc). 2005;125(12):460-6.

4. Serra-Majem L1, Ribas Barba L, Aranceta Bartrina $J$, et al. Obesidad infantil y juvenil en España. Resultados del Estudio Enkid (1998-2000).Med Clin(Barc). 2003; 121: 725-32.

5. Aranceta J, Pérez Rodrigo C, Muñoz M. Perfil nutricional de los ancianos institucionalizados en España. En: Muñoz M, Aranceta J, Guijarro JL (eds). Libro blanco de la alimentación del anciano en España. Madrid: Panamericana; 2004.

6. Rankinen T, Zuberi A, Chagnon YC, et al. The Human Obesity Gene Map: The 2005 update. Obesity 2006; 14 (4): 529-644.

7. Frayling TM, Timpson NJ, Weedon MN, et al. A common variant in the FTO gene is associated with body mass index and predisposes to childhood and adult obesity. Science 2007. 316: 889-94,

8. Crawford DC, Nickerson DA. Definition and clinical importance of haplotypes. Annu Rev Med. 2005; 56: 303-20.

9. Klerk M, Verhoef P, Clarke R, et al. MTHFR 677C$\rightarrow \mathrm{T}$ polymorphism and risk of coronary heart disease: A Meta-analysis. JAMA. 2002; 288:2023.

10. Kohara K, Fujisawa M, Ando F, et al. MTHFR Gene Polymorphism as a Risk Factor for Silent Brain Infarcts and White Matter Lesions in the Japanese General Population: The NILS-LSA Study. Stroke. 2003; 34:1130.

11. Tsai, AW, Cushman, M, Tsai, MY, et al. Serum homocysteine, thermolabile variant of methylene tetrahydrofolate reductase (MTHFR), and venous thromboembolism: Longitudinal Investigation of Thromboembolism Etiology (LITE). Am J Hematol. $2003 ; 72: 192$.

12. Bezemer, ID, Doggen, CJ, Vos, HL, Rosendaal, FR. No Association Between the Common
MTHFR 677C->T Polymorphism and Venous Thrombosis: Results From the MEGA Study. Arch Intern Med. 2007; 167:497.

13. Kupferminc MJ, Eldor A, Steinman N, et al. Increased frequency of genetic thrombophilia in women with complications of pregnancy. N Engl J Med. 1999; 340:9.

14. Altomare I, Adler A, Aledort LM. The 5, 10 methylenetetrahydrofolate reductase C677T mutation and risk of fetal loss: a case series and review of the literature. Thromb J. 2007, 5:17 (epub 17-10-2007).

15. Homocysteine Lowering Trialists' Collaboration. Lowering blood homocysteine with folic acid based supplements: meta-analysis of randomized trials. BMJ. 1998; 316:894-8.

16. Wald, DS, Bishop, L, Wald, NJ, et al. Randomized trial of folic acid supplementation and serum homocysteine levels. Arch Intern Med. 2001; 161:695.

17. Zoungas S, McGrath BP, Branley P, et al. Cardiovascular morbidity and mortality in the Atherosclerosis and Folic Acid Supplementation Trial (ASFAST) in chronic renal failure: a multicenter, randomized, controlled trial. J Am Coll Cardiol. 2006; 47:1108.

18. Bazzano LA, Reynolds K, Holder KN, He J. Effect of folic acid supplementation on risk of cardiovascular diseases: a meta-analysis of randomized controlled trials. JAMA. 2006; 296:2720.

19. den Heijer M, Willems HP, Blom HJ, et al. Homocysteine lowering by $\mathrm{B}$ vitamins and the secondary prevention of deep vein thrombosis and pulmonary embolism: A randomized, placebocontrolled, double-blind trial. Blood. 2007; 109:139.

20. Ordovas JM. Gene interactions with diet influence the risk of cardiovascular disease. Am J Clin Nutr. 2006; 83(suppl): 443S-6S.

21. Zeisel HS. Nutrigenomics and metabolomics will change clinical nutrition and public health practice: insights from studies on dietary requierements for choline, Am J Clin Nutr. 2007; 86; 542-8.

22. Sessler AM, Ntambi JM. Polyunsaturated fatty acid regulation of gene expression. J Nutr .1998;128:923-6.

23. Fernández ML, West KL. Mechanisms by which Dietary Fatty Acids Modulate Plasma Lipids. J Nutr. 2005; 135:2075-8. 
24. Fernández-Quintela A, Churruca I, Puy Portillo M. The role of dietary fat in adipose tissue metabolism. Pub Health Nutr. 2007; 10 (10A): 112631.

25. Clarke SD, Gasperikova D, Nelson C, et al.Fatty acid regulation of gene expression: a genomic explanation for the benefits of the mediterranean diet. Ann N Y Acad Sci 2002;967:283-98.

26. Pariza MW, Park Y, Cook ME. The biologically active isomers of conjugated linoleic acid. Prog Lipid Res. 2001;40(4):283-98.

27. House RL, Cassady JP, Eisen EJ, et al. Conjugated linoleic acid evokes de-lipidation through the regulation of genes controlling lipid metabolism in adipose and liver tissue. Obes Rev. 2005;6(3):247-58.

28. Tricon S, Burdge GC, Kew S, et al. Opposing effects of cis- 9 , trans-11 and trans-10, cis- 12 conjugated linoleic acid on blood lipids in healthy humans. Am J Clin Nutr. 2004;80(3):614-20.

29. Tricon S, Burdge GC, Jones EL, et al. Effects of dairy products naturally enriched with cis- 9 , trans11 conjugated linoleic acid on the blood lipid profile in healthy middle-aged men. Am J Clin Nutr. 2006;83(4):744-53.

30. Terpstra AH. Effect of conjugated linoleic acid on body composition and plasma lipids in humans: an overview of the literature. Am J Clin Nutr. 2004;79(3):352-61.

31. Choi Y, Kim YC, Han YB, et al. The trans-10,cis12 isomer of conjugated linoleic acid downregulates stearoyl-CoA desaturase 1 gene expression in 3T3-L1 adipocytes. J Nutr. 2000;130(8):1920-4.

32. Park Y, Storkson JM, Ntambi JM, et al. Inhibition of hepatic stearoyl-CoA desaturase activity by trans-10, cis-12 conjugated linoleic acid and its derivatives. Biochim Biophys Acta 2000;1486(23):285-92.

33. Park Y, Storkson JM, Albright KJ, et al. Evidence that the trans-10,cis-12 isomer of conjugated linoleic acid induces body composition changes in mice. Lipids. 1999 Mar;34(3):235-41.

34. Brown JM, Boysen MS, Chung S, et al. Conjugated linoleic acid (CLA) induces human adipocyte delipidation: autocrine/paracrine regulation of MEK/ERK signaling by adipocytokines. J Biol Chem. 2004; 279: 26735-47.

35. Riserus U, Smedman A, Basu S, Vessby B. Metabolic effects of conjugated linoleic acid in humans: the Swedish experience. Am J Clin Nutr. 2004; 79: 1146S-1148S.

36. Riserus U, Vessby B, Arner P, Zethelius B. Supplementation with trans10cis12-conjugated linoleic acid induces hyperproinsulinaemia in obese men: close association with impaired insulin sensitivity. Diabetologia. 2004;47(6):1016-9.

37. Moloney F, Yeow TP, Mullen A, et al. Conjugated linoleic acid supplementation, insulin sensitivity, and lipoprotein metabolism in patients with type 2 diabetes mellitus. Am J Clin Nutr. 2004; 80(4):887-95.

38. Moloney F, Toomey S, Noone E, et al. Antidiabetic Effects of cis-9, trans-11-Conjugated Linoleic Acid May Be Mediated via Anti-Inflammatory Effects in White Adipose Tissue. Diabetes. 2007; 56:574-582.

39. Poirier H, Shapiro JS, Kim RJ, Lazar MA. Nutritional Supplementation With trans-10, cis12-Conjugated Linoleic Acid Induces Inflammation of White Adipose Tissue. Diabetes. 2006; 55 : 1634-41.

40. Silveira MB, Carraro R, Monereo S, Tébar J. Conjugated linoleic acid (CLA) and obesity. Pub Health Nutr. 2007; 10 (10A): 1181-6.

41. Morgan HD, Sutherland HG, Martin DI, Whitelaw E. Epigenetic inheritance at the agouti locus in the mouse. Nat Genet. 1999; 23:314-8.

42. L.H. Lumey, Decreased birthweights in infants after maternal in utero exposure to the Dutch famine of 1944-1945. Paediatr Perinat Epidemiol. 1992; 6: 240-53.

43. Barker DJ. Fetal growth and adult disease. Br J Obstet Gynaecol. 1992;99:275-6.

44. Hales CN, Barker DJ.The thrifty phenotype hypothesis. Br Med Bull. 2001;60:5-20.

45. Barker DJ. The fetal origins of diseases of old age. Eur J Clin Nutr. 1992;46(suppl):S3-9.

46. Osmond C, Barker DJ, Winter PD, et al. Early growth and death from cardiovascular disease in women. BMJ. 1993;307:1519-24.

47. Barker DJ, Osmond C, Simmonds SJ, Wield GA. The relation of small head circumference and thinness at birth to death from cardiovascular disease in adult life. BMJ. 1993;306:422-6.

48. Rich-Edwards J, Stampfer M, Manson J, et al. Birthweight, breastfeeding, and the risk of coro- 
nary heart disease in the Nurses' Health Study. Am J Epidemiol. 1995;141:S78.

49. Frankel S, Elwood P, Sweetnam P, et al. Birthweight, adult risk factors and incident coronary heart disease: the Caerphilly Study. Public Health. 1996;110:139-43.

50. Leon DA, Koupilova I, Lithell HO, et al. Failure to realise growth potential in utero and adult obesity in relation to blood pressure in 50 year old Swedish men. BMJ. 1996;312:401-6.

51. Ravelli GP, Stein ZA, Susser MW. Obesity in young men after famine exposure in utero and early infancy. N Engl J Med. 1976;295:349-53.

52. Gluckman PD, Hanson MA.Living with the past: evolution, development and patterns of disease. Science. 2004; 305:1773-1776.

53. Hales CN, Barker DJ, Clark PM, et al. Fetal and infant growth and impaired glucose tolerance at age 64. BMJ. 1991;303:1019-22.

54. Phipps K, Barker DJ, Hales CN, et al. Fetal growth and impaired glucose tolerance in men and women. Diabetologia. 1993;36:225-8.

55. Phillips DI, Barker DJ, Hales CN, et al. Thinness at birth and insulin resistance in adult life. Diabetologia. 1994;37:150-4.

56. Vickers MH, Breier BH, Cutfield WS, et al. Fetal origins of hyperphagia, obesity and hypertension and its postnatal amplification by hypercaloric nutrition. Am J Physiol. 2000; 279:E83-E87.

57. Gluckman PD, Hanson MA, Spencer HG. Predictive adaptive responses and human evolution. Trends Ecol Evol. 2005; 20 (10): 527-33.

58. Rickard IJ, Lummaa V. The Predictive Adaptive Response and metabolic syndrome: Challenges for the hypothesis. Trends Endocrinol Metab. 2007; 18 (3): 94-9.

59. Pinto S, Roseberry AG, Liu H, et al. Rapid rewiring of arcuate nucleus feeding circuits by leptin. Science. 2004; 304:110 -115.

60. Bouret SG, Draper SJ, Simerly RB. Trophic action of leptin on hypothalamic neurons that regulate feeding. Science. 2004; 304:108 -110.

61. Islam M, Sjo“holm Å, Emilsson V. Fetal pancreatic islets express functional leptin receptors and leptin stimulates proliferation of fetal islet cells. Int J Obes Relat Metab Disord. 2000, 24:1246-53.
62. Vickers MH, Gluckman PD, Coveny AH, et al. Neonatal Leptin Treatment Reverses Developmental Programming. Endocrinology. 2005;146: 4211-6.

63. Miralles O, Sánchez J, Palou A, Picó C. A Physiological Role of Breast Milk Leptin in Body Weight Control in Developing Infants. Obesity. 2006; 14:-1371-77.

64. Uysal FK, Onal EE, Aral YZ, et al. Breast milk leptin: its relationship to maternal and infant adiposity. Clin Nutr. 2002;21:157-60.

65. Ucar B, Kirel B, Bor O, et al. Breast milk leptin concentrations in initial and terminal milk samples: relationships to maternal and infant plasma leptin concentrations, adiposity, serum glucose, insulin, lipid and lipoprotein levels. J Pediatr Endocrinol Metab. 2000;13:149 -56.

66. Kramer MS. Do breast-feeding and delayed introduction of solid foods protect against subsequent obesity? J Pediatr. 1981;98:883-7.

67. von Kries R, Koletzko B, Sauerwald T, et al. Breast feeding and obesity: cross sectional study. BMJ. 1999;319:147-50.

68. Armstrong J, Reilly JJ. Breastfeeding and lowering the risk of childhood obesity. Lancet. 2002;359:2003- 4 .

69. Hediger ML, Overpeck MD, Kuczmarski RJ, Ruan WJ. Association between infant breastfeeding and overweight in young children. JAMA. 2001;285:2453-60.

70. Li L, Parsons TJ, Power C. Breast feeding and obesity in childhood: cross sectional study. BMJ. 2003;327:904 -5.

71. Kaati G, Bygren LO, Edvinssons S. Cardiovascular and diabetes mortality determined by nutrition during parents' and grandparents' slow growth period. Eur J Hum Genet. 2002; 10:682-8.

72. Baker J, Liu JP, Robertson EJ, Efstratiadis A. Role of insulinlike growth factors in embryonic and postnatal growth. Cell. 1993; 75:73-82.

73. Constancia M, Hemberger M, Hughes J, et al. Placental-specific IGF-II is a major modulator of placental and fetal growth. Nature. 2002; 417: 945-8.

74. Pietiläinen K, Rissanen A, Laamanen M, et al. Growth Patterns in Young Adult Monozygotic Twin Pairs Discordant and Concordant for Obesity. Twin Res. 2005; 7 (5): 421-429. 
75. Kaiser J. Developmental biology. Endocrine disrupters trigger fertility problems in multiple generations. Science. 2005; 308:1391-2.

76. Feil R. Environmental and nutritional effects on the epigenetic regulation of genes Mutat Res. 2006; 600: 46-57.

77. Pembrey ME. Time to take epigenetic inheritance seriously. Eur J Hum Genet. 2002; 10:669-71.

78. Yu YH, Zhu H. Chronological changes in metabolism and functions of cultured adipocytes: a hypothesis for cell aging in mature adipocytes. AJPEndo 2004; 286:402-10.

79. Silveira MB, Carraro R, Gómez-Pan A. El aparato digestivo como órgano endocrino: nuevas perspectivas en el tratamiento de la obesidad. Med Clín (Barc). 2006; 127: 300-5.
80. Slawik M, Vidal-Puig AJ. Lipotoxicity, overnutrition and energy metabolism in aging. Aging Res Rev. 2006; 5: 144-164.

81. Unger RH, Orci L. Lipoapoptosis: its mechanism and its diseases. Biochim Biophys Acta. 2002;1585: 202-212.

82. Shulman GI. Cellular mechanisms of insulin resistance in humans. Am J Cardiol. 1999; 84: 3J-10J.

83. Recasens M, Ricart W, Fernandez-Real JM. Obesidad e inflamación. Rev Med Univ Navarra 2004;48(2):49-54.

84. Ruiz-Torres A, Lozano R, Melón J, et al. On how insulin may influence ageing and become atherogenic throughout IGF-1 receptor pathway: In vitro studies with human vascular smooth muscle cells. Gerontology. 2005; 51:225-230. 
